\title{
Multifucosylated alpha-1-acid glycoprotein as a novel marker for hepatocellular carcinoma
}

Kazuhiro Tanabe ${ }^{1 *}$, Kae Kitagawa ${ }^{2}$, Nozomi Kojima², Sadayo Iijima ${ }^{3}$

${ }^{1}$ Advanced Technology Center, Medical Solution Segment, LSI Medience Corporation, Tokyo, Japan.

${ }^{2}$ Biotechnology Laboratory, Mitsubishi Chemical Group Science and Technology Research Center, Inc., Yokohama, Japan

${ }^{3}$ International Sales Department, LSI Medience Corporation, Tokyo, Japan

\section{Table of Contents:}

Page S-2: Figure S-1. A1711 identification using MS/MS and Mascot search Page S-3: Figure S-2. Spectrum comparison between A1711 and glycopeptides of purified AGP 


\section{$\left\{\begin{array}{l}\{\text { MATRIXNE }\} \\ \{\text { Mascot Search Results }\end{array}\right.$}

\section{Peptide View}

MS/MS Fragmentation of SVQEIQATFFYFTPNKTEDTIFLR

Found in A1AG1_HUMAN, Alpha-1-acid glycoprotein 1 OS=Homo sapiens GN=ORM1 PE=1 SV=1

Match to Query 856: 2894.845233 from(965.955687,3+) rtinseconds(1634.634) index(774) Title: MSMS1.d, MS/MS of $965.95568743+$ at 27.2439 mins

Data file F: $\backslash Q-T O F 3$ DATA $110712 \_$MCHMa1711_MSMS $\backslash M S M S 1 . m g f$

Click mouse within plot area to zoom in by factor of two about that point

Or, Plot from 0

to 2600

Da Full range

Label all possible matches $\odot$ Label matches used for scoring $\odot$

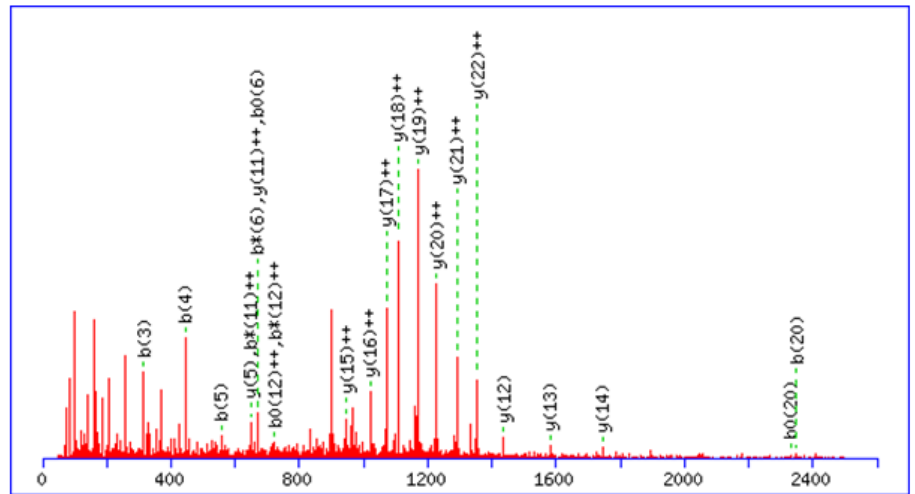

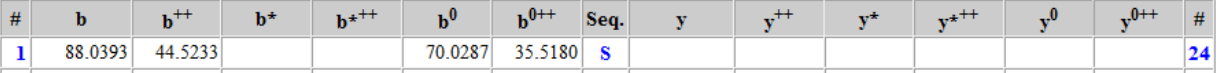
\begin{tabular}{|l|l|l|l|l|l|l|l|l|l|l|l|l|l|l|}
\hline $\mathbf{2}$ & 187.1077 & 94.0575 & & 169.0972 & 85.0522 & $\mathbf{V}$ & 2809.4083 & 1405.2078 & 2792.3818 & 1396.6945 & 2791.3978 & 1396.2025 & $\mathbf{2 3}$ \\
\hline
\end{tabular}

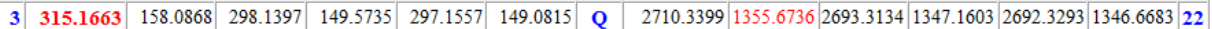

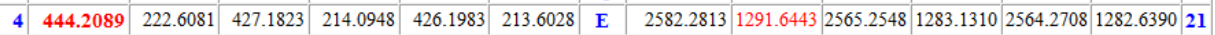

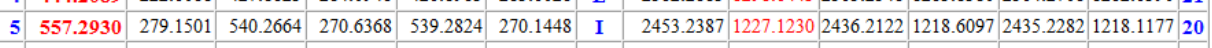
\begin{tabular}{|c|c|c|c|c|c|c|c|c|c|c|c|c|c|c|}
\hline $\mathbf{6}$ & 685.3515 & 343.1794 & 668.3250 & 334.6661 & 667.3410 & 334.1741 & $\mathbf{Q}$ & 2340.1547 & 1170.5810 & 2323.1281 & 1162.0677 & 2322.1441 & 1161.5757 & 19 \\
\hline
\end{tabular}

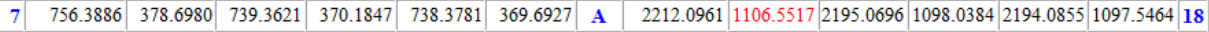
\begin{tabular}{|c|c|c|c|c|c|c|c|c|c|c|c|c|c|c|}
\hline $\mathbf{8}$ & 857.4363 & 429.2218 & 840.4098 & 420.7085 & 839.4258 & 420.2165 & T & 2141.0590 & 1071.0331 & 2124.0324 & 1062.5199 & 2123.0484 & 1062.0278 & 17
\end{tabular} \begin{tabular}{|l|l|l|l|l|l|l|l|l|l|l|l|l|l|l|l|}
$\mathbf{9}$ & 1004.5047 & 502.7560 & 987.4782 & 494.2427 & 986.4942 & 493.7507 & F & 2040.0113 & 1020.5093 & 2022.9848 & 1011.9960 & 2022.0007 & 1011.5040 & 16 \\
\hline
\end{tabular} \begin{tabular}{|l|l|l|l|l|l|l|l|l|l|l|l|l|l|l|l|}
\hline 10 & 1151.5732 & 576.2902 & 1134.5466 & 567.7769 & 1133.5626 & 567.2849 & F & 1892.9429 & 946.9751 & 1875.9163 & 938.4618 & 1874.9323 & 937.9698 & 15 \\
\hline
\end{tabular} \begin{tabular}{|l|l|l|l|l|l|l|l|l|l|l|l|l|l|l|}
11 & 1314.6365 & 657.8219 & 1297.6099 & 649.3086 & 1296.6259 & 648.8166 & $\mathbf{Y}$ & 1745.8745 & 873.4409 & 1728.8479 & 864.9276 & 1727.8639 & 864.4356 & 14 \\
\hline
\end{tabular} \begin{tabular}{|l|l|l|l|l|l|l|l|l|l|l|l|l|l|l|l|}
12 & 1461.7049 & 731.3561 & 1444.6783 & 722.8428 & 1443.6943 & 722.3508 & F & 1582.8112 & 791.9092 & 1565.7846 & 783.3959 & 1564.8006 & 782.9039 & 13 \\
\hline
\end{tabular}

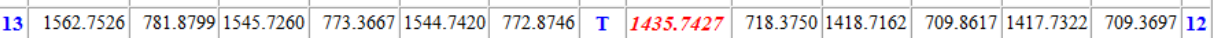
\begin{tabular}{|l|l|l|l|l|l|l|l|l|l|l|l|l|l|l|l|}
\hline 14 & 1659.8053 & 830.4063 & 1642.7788 & 821.8930 & 1641.7948 & 821.4010 & $\mathbf{P}$ & 1334.6951 & 667.8512 & 1317.6685 & 659.3379 & 1316.6845 & 658.8459 & $\mathbf{1 1}$ \\
\hline
\end{tabular} \begin{tabular}{|l|l|l|l|l|l|l|l|l|l|l|l|l|l|l|l|l|}
15 & 1774.8323 & 887.9198 & 1757.8057 & 879.4065 & 1756.8217 & 878.9145 & $\mathbf{N}$ & 1237.6423 & 619.3248 & 1220.6157 & 610.8115 & 1219.6317 & 610.3195 & $\mathbf{1 0}$ \\
\hline
\end{tabular} \begin{tabular}{|l|l|l|l|l|l|l|l|l|l|l|l|l|l|l|l|l|l|l|}
\hline 16 & 1902.9272 & 951.9673 & 1885.9007 & 943.4540 & 1884.9167 & 942.9620 & $\mathrm{~K}$ & 1122.6154 & 561.8113 & 1105.5888 & 553.2980 & 1104.6048 & 552.8060 & 9 \\
\hline
\end{tabular} \begin{tabular}{|l|l|l|l|l|l|l|l|l|l|l|l|l|l|l|l|}
\hline 17 & 2003.9749 & 1002.4911 & 1986.9484 & 993.9778 & 1985.9644 & 993.4858 & T & 994.5204 & 497.7638 & 977.4938 & 489.2506 & 976.5098 & 488.7585 & 8 \\
\hline
\end{tabular} \begin{tabular}{|l|l|l|l|l|l|l|l|l|l|l|l|l|l|l|l|l|}
\hline 18 & 2133.0175 & 1067.0124 & 2115.9910 & 1058.4991 & 2115.0070 & 1058.0071 & E & 893.4727 & 447.2400 & 876.4462 & 438.7267 & 875.4621 & 438.2347 & 7 \\
\hline
\end{tabular} \begin{tabular}{|l|l|l|l|l|l|l|l|l|l|l|l|l|l|l|l|}
\hline 19 & 2248.0445 & 1124.5259 & 2231.0179 & 1116.0126 & 2230.0339 & 1115.5206 & D & 764.4301 & 382.7187 & 747.4036 & 374.2054 & 746.4196 & 373.7134 & 6 \\
\hline
\end{tabular}

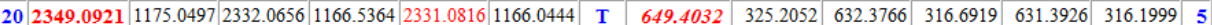
21 2462.17621231 .59172445 .14971223 .07852444 .16561222 .5805 | I

\begin{tabular}{l|l|l|l|l|l|l|l|l|l|l|l|l|l|l|l|l|l}
22 & 2609.2446 & 1305.1259 & 2592.2181 & 1296.6127 & 2591.2341 & 1296.1207 & F & \\
\hline
\end{tabular} \begin{tabular}{l|l|l|l|l|l|l|l|l|l|l|l|l}
23 & 2722.3287 & 1361.6680 & 2705.3021 & 1353.1547 & 2704.3181 & 1352.6627 & L & \\
\end{tabular}

24

$5483555 \quad 274.6814$ 5313289 2661681

\begin{tabular}{|l|l|l|l|l|l}
\hline 435.2714 & 218.1394 & 418.2449 & 209.626 \\
\hline
\end{tabular}

\begin{tabular}{|l|l|l|l|l}
288.2030 & 144.6051 & 271.1765 & 136.091
\end{tabular}

\begin{tabular}{|l|l|l|l|l|l|l|l|l}
175.1190 & 88.0631 & 158.0924 & 79.5498 \\
\hline
\end{tabular}
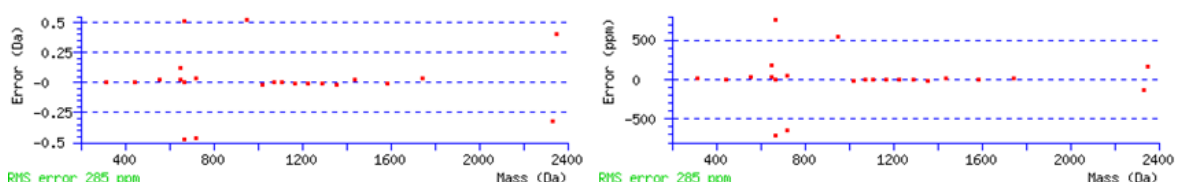

Figure S-1. A1711 identification using MS/MS and Mascot search

A1711 was isolated by liquid chromatography, followed by removing N-glycans by PNGaseF and analyzed by LCMS/MS. The data was analyzed by Mascot. 


\section{Peptide Sequence: SVQEIQATFFYFTPN * KTEDTIFLR}

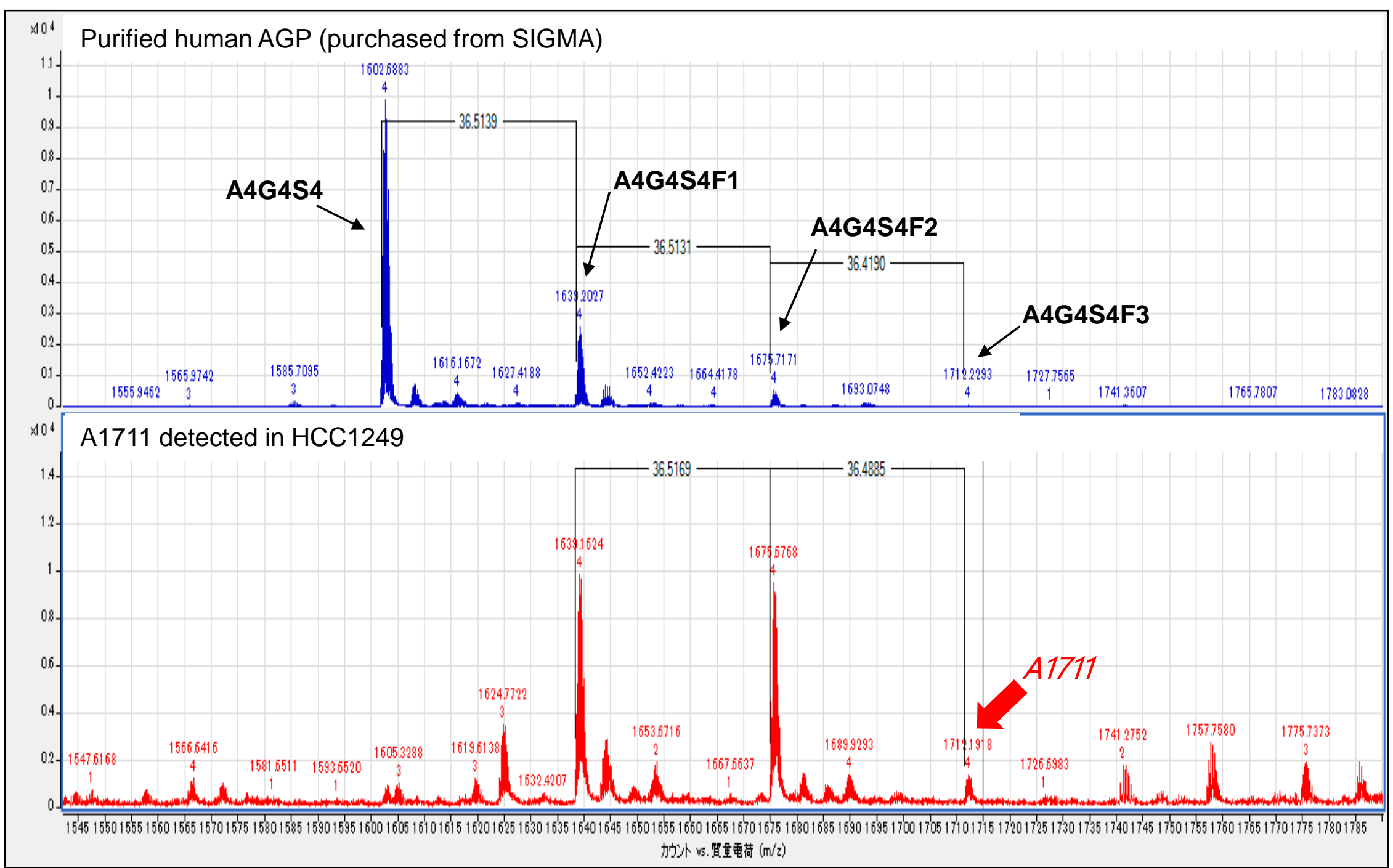

\section{Figure S-2. Spectrum comparison between A1711 and glycopeptides of purified AGP}

The tryptic digests of purified human AGP was analyzed LC-MS and compared with the spectrum of A1711. Upper: mass spectrum of tryptic digests of purified human AGP, Lower: the spectrum of A1711 in HCC patient 